\title{
Nonlocal initial and boundary value problems via fractional calculus with exponential singular kernel
}

\author{
Sotiris K. Ntouyas ${ }^{\mathrm{a}, \mathrm{b}}$, Jessada Tariboon ${ }^{\mathrm{c}, \mathrm{d}, *}$, Chalong Sawaddee ${ }^{\mathrm{e}}$ \\ ${ }^{a}$ Department of Mathematics, University of loannina, 45110 loannina, Greece. \\ ${ }^{b}$ Nonlinear Analysis and Applied Mathematics (NAAM)-Research Group, Department of Mathematics, Faculty of Science, King \\ Abdulaziz University, P. O. Box 80203, Jeddah 21589, Saudi Arabia. \\ ${ }^{c}$ Nonlinear Dynamic Analysis Research Center, Department of Mathematics, Faculty of Applied Science, King Mongkut's University of \\ Technology North Bangkok, Bangkok 10800, Thailand. \\ ${ }^{d}$ Centre of Excellence in Mathematics, CHE, Sri Ayutthaya Rd., Bangkok 10400, Thailand. \\ ${ }^{e}$ Department of Applied Mathematics and Statistics, Faculty of Sciences and Liberal Arts, Rajamangala University of Technology Isan, \\ Nakhon Ratchasima 30000, Thailand.
}

Communicated by N. Shahzad

\begin{abstract}
In this paper, we investigate the existence and uniqueness of solutions for nonlocal initial and boundary value problems of exponential fractional differential equations, by applying standard fixed point theorems. Enlightening examples are also presented.
\end{abstract}

Keywords: Exponential fractional integral, exponential fractional derivative, nonlocal initial value problems, nonlocal boundary value problems, fixed point theorems.

2010 MSC: 34A08, 34A12, 34B15.

(C)2018 All rights reserved.

\section{Introduction}

In recent years, the theory of fractional differential equations has been widely used in various fields, such as physics, mechanics, chemistry, engineering, etc., see $[10,13,15,16,19,20]$. Initial and boundary value problems of nonlinear fractional differential equations have been addressed by several researchers and has been growing rapidly, see [1-6, 11, 17, 18, 21, 22, 24, 25] and the references cited therein.

In [13, Section 5.2] fractional integrals and fractional derivatives of a function with respect to another function were defined as the following.

\footnotetext{
*Corresponding author

Email addresses: sntouyas@uoi.gr (Sotiris K. Ntouyas), jessada.t@sci.kmutnb.ac.th (Jessada Tariboon), chalong07@yahoo.com (Chalong Sawaddee)
}

doi: $10.22436 /$ jnsa.011.09.01

Received: 2017-10-05 Revised: 2018-05-04 Accepted: 2018-05-05 
For an increasing and positive monotone function $\mathrm{g}:(\mathrm{a}, \mathrm{b}) \rightarrow \mathbb{R}(-\infty \leqslant \mathrm{a}<\mathrm{b} \leqslant \infty)$ having a continuous derivative $g^{\prime}(x)$ on $(a, b)$, the left and right-sided fractional integrals of order $\alpha, \mathfrak{R}(\alpha)>0$, of a function $f$ with respect to another function $g$ on $[a, b]$ are defined by

$$
\left(I_{a+, g}^{\alpha} f\right)(x)=\frac{1}{\Gamma(\alpha)} \int_{a}^{x}[g(x)-g(s)]^{\alpha-1} g^{\prime}(s) f(s) d s, \quad x>a,
$$

and

$$
\left(I_{b-, g}^{\alpha} f\right)(x)=\frac{1}{\Gamma(\alpha)} \int_{x}^{b}[g(s)-g(x)]^{\alpha-1} g^{\prime}(s) f(s) d s, \quad x<b,
$$

respectively. When $a=0$ and $b=\infty$, then

$$
\left(I_{0+, g}^{\alpha} f\right)(x)=\frac{1}{\Gamma(\alpha)} \int_{0}^{x}[g(x)-g(s)]^{\alpha-1} g^{\prime}(s) f(s) d s, \quad x>0,
$$

and

$$
\left(I_{-, g}^{\alpha} f\right)(x)=\frac{1}{\Gamma(\alpha)} \int_{x}^{\infty}[g(s)-g(x)]^{\alpha-1} g^{\prime}(s) f(s) d s, \quad x>0,
$$

while, for $a=-\infty$ and $b=\infty$ then

$$
\left(I_{+, g}^{\alpha} f\right)(x)=\frac{1}{\Gamma(\alpha)} \int_{-\infty}^{x}[g(x)-g(s)]^{\alpha-1} g^{\prime}(s) f(s) d s, \quad x \in \mathbb{R},
$$

and

$$
\left(I_{-, g}^{\alpha} f\right)(x)=\frac{1}{\Gamma(\alpha)} \int_{x}^{\infty}[g(s)-g(x)]^{\alpha-1} g^{\prime}(s) f(s) d s, \quad x \in \mathbb{R} .
$$

It is well known that if $g(x)=x$ then all results in (1.1)-(1.6) are reduced to the Riemann-Liouville fractional integrals, and if $g(x)=\log _{e} x$ then the above formulas (1.1)-(1.6) are reduced to the Hadamard fractional integrals. In the case when $g(x)=e^{x}$ we have new kind of fractional calculus which is based on exponential fractional integrals defined as the following.

Definition 1.1. The exponential left and right-sided fractional integral of a function $f: \mathbb{R} \rightarrow \mathbb{R}$ of order $\alpha \geqslant 0$ are defined by

$$
{ }^{e} I_{a+}^{\alpha} f(x)=\frac{1}{\Gamma(\alpha)} \int_{a}^{x}\left(e^{x}-e^{t}\right)^{\alpha-1} f(t) e^{t} d t, \quad x>a, \quad \alpha>0,
$$

and

$$
{ }^{e} \mathrm{I}_{\mathrm{b}-}^{\alpha} f(x)=\frac{1}{\Gamma(\alpha)} \int_{x}^{b}\left(e^{x}-e^{t}\right)^{\alpha-1} f(t) e^{t} d t, \quad x<b, \quad \alpha>0,
$$

respectively and ${ }^{e} I_{(\cdot)}^{0} f(x)=f(x)$.

If $a=0$ and $b=\infty$, then

$$
{ }^{e} I_{0+}^{\alpha} f(x)=\frac{1}{\Gamma(\alpha)} \int_{0}^{x}\left(e^{x}-e^{t}\right)^{\alpha-1} f(t) e^{t} d t, \quad x>0, \quad \alpha>0,
$$

and

$$
{ }^{e} \mathrm{I}_{-}^{\alpha} f(x)=\frac{1}{\Gamma(\alpha)} \int_{x}^{\infty}\left(e^{x}-e^{t}\right)^{\alpha-1} f(t) e^{t} d t, \quad x \in \mathbb{R}, \quad \alpha>0,
$$

while, if $a=-\infty$, then

$$
{ }^{e} I_{+}^{\alpha} f(x)=\frac{1}{\Gamma(\alpha)} \int_{-\infty}^{x}\left(e^{x}-e^{t}\right)^{\alpha-1} f(t) e^{t} d t, \quad x \in \mathbb{R}, \quad \alpha>0 .
$$


Let $A C_{e}^{n}[a, b]=\left\{g:[a, b] \rightarrow \mathbb{C}:{ }^{e} D^{n-1} g(x) \in A C[a, b],{ }^{e} D=e^{-x} \frac{d}{d x}\right\}$. For $f \in A C_{e}^{n}[a, b]$, where $-\infty \leqslant \mathrm{a}<\mathrm{b} \leqslant \infty$, we define the exponential fractional derivatives of Riemann-Liouville and Caputo types as follow.

Definition 1.2. The exponential left and right-sided fractional derivatives of Riemann-Liouville type of order $\alpha \geqslant 0$ for a function $f: \mathbb{R} \rightarrow \mathbb{R}$ are defined by

$$
\begin{aligned}
& { }^{e} D_{a+}^{\alpha} f(x)=\frac{1}{\Gamma(n-\alpha)}\left(e^{-x} \frac{d}{d x}\right)^{n} \int_{a}^{x}\left(e^{x}-e^{t}\right)^{n-\alpha-1} f(t) \frac{d t}{e^{-t}}, \quad x>a, \quad \alpha>0, \\
& { }^{e} D_{b-}^{\alpha} f(x)=\frac{1}{\Gamma(n-\alpha)}\left(-e^{-x} \frac{d}{d x}\right)^{n} \int_{x}^{b}\left(e^{x}-e^{t}\right)^{n-\alpha-1} f(t) \frac{d t}{e^{-t}}, \quad x<b, \quad \alpha>0 .
\end{aligned}
$$

If $a=0$ and $b=\infty$, then

$$
\begin{aligned}
{ }^{e} D_{0+}^{\alpha} f(x) & =\frac{1}{\Gamma(n-\alpha)}\left(e^{-x} \frac{d}{d x}\right)^{n} \int_{0}^{x}\left(e^{x}-e^{t}\right)^{n-\alpha-1} f(t) \frac{d t}{e^{-t}}, \quad x>0, \quad \alpha>0, \\
{ }^{e} D_{-}^{\alpha} f(x) & =\frac{1}{\Gamma(n-\alpha)}\left(-e^{-x} \frac{d}{d x}\right)^{n} \int_{x}^{\infty}\left(e^{x}-e^{t}\right)^{n-\alpha-1} f(t) \frac{d t}{e^{-t}}, \quad x \in \mathbb{R}, \quad \alpha>0,
\end{aligned}
$$

while, if $a=-\infty$, then

$$
{ }^{e} D_{+}^{\alpha} f(x)=\frac{1}{\Gamma(n-\alpha)}\left(e^{-x} \frac{d}{d x}\right)^{n} \int_{-\infty}^{x}\left(e^{x}-e^{t}\right)^{n-\alpha-1} f(t) \frac{d t}{e^{-t}}, \quad x \in \mathbb{R}, \quad \alpha>0,
$$

and ${ }^{e} D^{0} f(x)=f(x)$, where $n$ is the smallest integer greater than or equal to $\alpha$.

Definition 1.3. The exponential left and right-sided fractional derivatives of Caputo type of order $\alpha \geqslant 0$ for a function $f: \mathbb{R} \rightarrow \mathbb{R}$ is defined as

$$
{ }_{C}^{e} D_{a+}^{\alpha} f(x)=\frac{1}{\Gamma(n-\alpha)} \int_{a}^{x}\left(e^{x}-e^{t}\right)^{n-\alpha-1}\left(e^{-t} \frac{d}{d t}\right)^{n} f(t) \frac{d t}{e^{-t}}, \quad x>a, \quad \alpha>0,
$$

and

$$
{ }_{C}^{e} D_{b-}^{\alpha} f(x)=\frac{1}{\Gamma(n-\alpha)} \int_{x}^{b}\left(e^{x}-e^{t}\right)^{n-\alpha-1}\left(e^{-t} \frac{d}{d t}\right)^{n} f(t) \frac{d t}{e^{-t}}, \quad x<b, \quad \alpha>0,
$$

and ${ }_{C}^{e} D_{(\cdot)}^{0} f(x)=f(x)$, where $n$ is the smallest integer greater than or equal to $\alpha$. The cases $a=0, b=\infty$ and $a=-\infty, b=\infty$ are defined analogously as in Definition 1.2.

Next, we state some important properties of exponential fractional calculus and also use the notations ${ }^{e} \mathrm{I}_{\mathrm{a}}^{\alpha},{ }^{e} \mathrm{D}_{\mathrm{a}}^{\alpha}$, and ${ }_{\mathrm{C}}^{e} \mathrm{D}_{\mathrm{a}}^{\alpha}$ for the left-side exponential fractional integral and derivatives of Riemann-Liouville and Caputo, respectively, which have the lower limit at a point $a$, where $a \in \mathbb{R} \cup\{-\infty\}$ as

$$
\begin{aligned}
{ }^{e} I_{a}^{\alpha} f(x) & =\frac{1}{\Gamma(\alpha)} \int_{a}^{x}\left(e^{x}-e^{t}\right)^{\alpha-1} f(t) e^{t} d t \\
{ }^{e} D_{a}^{\alpha} f(x) & =\frac{1}{\Gamma(n-\alpha)}\left(e^{-x} \frac{d}{d x}\right)^{n} \int_{a}^{x}\left(e^{x}-e^{t}\right)^{n-\alpha-1} f(t) \frac{d t}{e^{-t}}
\end{aligned}
$$

and

$$
{ }_{C}^{e} D_{a}^{\alpha} f(x)=\frac{1}{\Gamma(n-\alpha)} \int_{a}^{x}\left(e^{x}-e^{t}\right)^{n-\alpha-1}\left(e^{-t} \frac{d}{d t}\right)^{n} f(t) \frac{d t}{e^{-t}}
$$

The fractional integration and differentiation of the power function $\left(e^{x}-e^{a}\right)^{\beta}$ is given below. 
Property 1.4. If $\alpha, \beta>0$, then following relations hold for

$$
\begin{aligned}
{ }^{e} \mathrm{I}_{\mathrm{a}}^{\alpha}\left(e^{x}-e^{\mathrm{a}}\right)^{\beta} & =\frac{\Gamma(\beta+1)}{\Gamma(\alpha+\beta+1)}\left(e^{x}-e^{a}\right)^{\alpha+\beta}, \\
{ }^{e} \mathrm{D}_{\mathrm{a}}^{\alpha}\left(e^{x}-e^{a}\right)^{\beta} & =\frac{\Gamma(\beta+1)}{\Gamma(\beta-\alpha+1)}\left(e^{x}-e^{a}\right)^{\beta-\alpha}, \quad x \in[a, b] .
\end{aligned}
$$

Theorem 1.5 (Semigroup property). If $\alpha, \beta>0$, then the equations

$$
{ }^{e} \mathrm{I}_{\mathrm{a}}^{\alpha}\left({ }^{e} \mathrm{I}_{\mathrm{a}}^{\beta} \mathrm{f}\right)(x)={ }^{e} \mathrm{I}_{\mathrm{a}}^{\beta}\left({ }^{e} \mathrm{I}_{\mathrm{a}}^{\alpha} \mathrm{f}\right)(x)={ }^{e} \mathrm{I}_{\mathrm{a}}^{\alpha+\beta} \mathrm{f}(x),
$$

are satisfied for all $x \in[a, b]$.

The relation between exponential fractional derivatives of Riemann-Liouville and Caputo types is given by

$$
{ }_{C}^{e} D_{a}^{\alpha} f(x)={ }^{e} D_{a}^{\alpha}\left[f(t)-\sum_{k=0}^{n-1} \frac{{ }^{e} D^{k} f(a)}{k !}\left(e^{t}-e^{a}\right)^{k}\right](x),
$$

where ${ }^{e} \mathrm{D}=e^{-x} \frac{\mathrm{d}}{\mathrm{dx}}$.

Theorem 1.6. If $0<\beta<\alpha$, and $1 \leqslant p<\infty$, then for $f \in \mathrm{L}^{\mathrm{p}}(\mathrm{a}, \mathrm{b})$,

$$
{ }^{e} D_{a}^{\beta}\left({ }^{e} I_{a}^{\alpha} f\right)(x)={ }^{e} I_{a}^{\alpha-\beta} f(x) \text { and }{ }_{C}^{e} D_{a}^{\beta}\left({ }^{e} I_{a}^{\alpha} f\right)(x)={ }^{e} I_{a}^{\alpha-\beta} f(x) \text {. }
$$

In addition, we have

$$
{ }^{e} D_{a}^{\alpha}\left({ }^{e} I_{a}^{\alpha} f\right)(x)=f(x) \text { and } \quad{ }_{C}^{e} D_{a}^{\alpha}\left({ }^{e} I_{a}^{\alpha} f\right)(x)=f(x) .
$$

Theorem 1.7. Let $\alpha>0$ and $n=[\alpha]+1$, then the following formulas are true:

(i) ${ }^{e} I_{a}^{\alpha}\left({ }^{e} D_{a}^{\alpha} f\right)(x)=f(x)-\sum_{j=1}^{n} \frac{\left(e^{x}-e^{a}\right)^{\alpha-j}}{\Gamma(\alpha-j+1)}{ }^{e} D^{n-j}\left({ }^{e} I_{a}^{n-\alpha} f\right)(a)$;

(ii) ${ }^{e} I_{a}^{\alpha}\left({ }_{c}^{e} D_{a}^{\alpha} f\right)(x)=f(x)-\sum_{j=0}^{n-1} \frac{\left(e^{x}-e^{a}\right)^{j}}{j !}{ }^{j} D^{j} f(a)$.

Recently in [23] we studied initial and boundary value problems for exponential fractional differential equations. Here we extend the results in [23] to cover nonlocal initial and boundary value problems for exponential fractional differential equations. We consider a variety of initial and boundary value problems of exponential fractional differential equations. Existence and uniqueness results are obtained by using standard fixed point theorems, such as Banach's contraction mapping principle, Krasnoselskii's fixed point theorem, and Leray-Schauder nonlinear alternative.

\section{Main results}

We study existence and uniqueness results for nonlocal initial and boundary value problems for exponential fractional differential equations.

\subsection{Nonlocal initial value problems}

In this subsection we consider the following initial value problem for fractional differential equations with nonlocal initial conditions

$$
\left\{\begin{array}{l}
{ }_{C}^{e} D_{0}^{q} x(t)=f(t, x(t)), \quad 0<t<T, 0<q \leqslant 1, \\
x(0)+\sum_{j=1}^{m} \gamma_{j} x\left(t_{j}\right)=0,
\end{array}\right.
$$

where ${ }_{C}^{e} D_{0}^{q}$ is the exponential derivative of Caputo type of order $q, f:[0, T] \times \mathbb{R} \rightarrow \mathbb{R}, t_{j}, j=1,2, \ldots, m$ 
are given points with $0 \leqslant t_{1} \leqslant \ldots \leqslant t_{m} \leqslant T$, and $\gamma_{j}$ are real numbers with

$$
1+\sum_{j=1}^{m} \gamma_{j} \neq 0
$$

The problem (2.1) was studied in [8] for $q=1$, in [7] in time scales setting, and in [9] for Caputo fractional differential equations. Here we extend the results of [7-9] to the exponential derivative of Caputo type.

Let $X=C([0, T], \mathbb{R})$ denote the Banach space of all continuous functions from $[0, T]$ into $\mathbb{R}$ with the norm

$$
\|x\|=\sup _{t \in[0, T]}|x(t)| .
$$

The notation $L^{1}([0, T], \mathbb{R})$ denotes the Banach space of measurable functions $x:[0, T] \longrightarrow \mathbb{R}$ which are Lebesgue integrable and normed by

$$
\|x\|_{\mathrm{L}^{1}}=\int_{0}^{\mathrm{T}}|x(\mathrm{t})| \mathrm{dt} \quad \text { for all } \quad x \in \mathrm{L}^{1}([0, \mathrm{~T}], \mathbb{R}) .
$$

In order to define the solution of the problem (2.1), we consider the following lemma.

Lemma 2.1. Assume that $1+\sum_{j=1}^{m} \gamma_{j} \neq 0$. For a given function $\rho \in X$, the unique solution of the nonlocal initial value problem

$$
\left\{\begin{array}{l}
{ }_{C}^{e} D_{0}^{q} x(t)=\rho(t), 0<t<T, 0<q \leqslant 1 \\
x(0)+\sum_{j=1}^{m} \gamma_{j} x\left(t_{j}\right)=0,0 \leqslant t_{1} \leqslant \cdots \leqslant t_{m} \leqslant T
\end{array}\right.
$$

is given by

$$
x(t)=\frac{1}{\Gamma(q)} \int_{0}^{t}\left(e^{t}-e^{s}\right)^{q-1} \rho(s) e^{s} d s-\frac{1}{1+\sum_{j=1}^{m} \gamma_{j}} \sum_{j=1}^{m} \gamma_{j} \frac{1}{\Gamma(q)} \int_{0}^{t_{j}}\left(e^{t_{j}}-e^{s}\right)^{q-1} \rho(s) e^{s} d s .
$$

Proof. For any constant $\xi \in \mathbb{R}$, we have

$$
x(\mathrm{t})=\frac{1}{\Gamma(\mathbf{q})} \int_{0}^{\mathrm{t}}\left(e^{\mathrm{t}}-e^{\mathrm{s}}\right)^{\mathrm{q}-1} \rho(\mathrm{s}) e^{\mathrm{s}} \mathrm{ds}-\xi
$$

Then we obtain

$$
x\left(t_{j}\right)=\frac{1}{\Gamma(q)} \int_{0}^{t_{j}}\left(e^{t_{j}}-e^{s}\right)^{q-1} \rho(s) e^{s} d s-\xi
$$

Applying the initial condition for (2.2), we find that

$$
\xi=\frac{1}{1+\sum_{j=1}^{m} \gamma_{j}} \sum_{j=1}^{m} \gamma_{j} \frac{1}{\Gamma(q)} \int_{0}^{t_{j}}\left(e^{t_{j}}-e^{s}\right)^{q-1} \rho(s) e^{s} d s .
$$

Substituting the value of $\xi$ in (2.4), we obtain the unique solution of (2.2) given by (2.3). This completes the proof.

We shall assume throughout the remainder of the paper that $1+\sum_{j=1}^{m} \gamma_{j} \neq 0$, and put, for brevity,

$$
\alpha=\left(1+\sum_{j=1}^{m} \gamma_{j}\right)^{-1}, A=1+|\alpha| \sum_{j=1}^{m}\left|\gamma_{j}\right|
$$


In view of Lemma 2.1, solutions of (2.1) are fixed points of the operator $\mathbf{F}: X \rightarrow X$, defined by

$$
(\mathbf{F x})(\mathrm{t})=\frac{1}{\Gamma(\mathrm{q})} \int_{0}^{t}\left(e^{\mathrm{t}}-e^{\mathrm{s}}\right)^{\mathrm{q}-1} f(s, x(s)) e^{s} \mathrm{~d} s-\alpha \sum_{j=1}^{m} \gamma_{j} \int_{0}^{t_{j}}\left(e^{t_{j}}-e^{s}\right)^{q-1} f(s, x(s)) e^{s} d s .
$$

Our first existence and uniqueness result is based on Banach's fixed point theorem.

Theorem 2.2. Assume that $\mathrm{f}:[0, \mathrm{~T}] \times \mathbb{R} \rightarrow \mathbb{R}$ is a continuous function and satisfies the assumption: $\left(A_{1}\right)|f(t, x)-f(t, y)| \leqslant L\|x-y\|, \quad \forall t \in[0, T], L>0, x, y \in \mathbb{R}$.

If

$$
L<\frac{\Gamma(q+1)}{A\left(e^{T}-1\right)^{q}}
$$

then the nonlocal initial value problem (2.1) has a unique solution on $[0, \mathrm{~T}]$.

Proof. In view of Lemma 2.1, solutions of (2.1) are fixed points of the operator $\mathbf{F}: X \rightarrow X$, defined by (2.5). Setting $\sup _{t \in[0, T]}|f(t, 0)|=M$ and choosing $r \geqslant \frac{A\left(e^{\top}-1\right)^{q} M}{\Gamma(q+1)-A\left(e^{T}-1\right)^{q} L}$, we show that $F B_{r} \subset B_{r}$, where $\mathrm{B}_{\mathrm{r}}=\{x \in X:\|x\| \leqslant r\}$. For $x \in \mathrm{B}_{\mathrm{r}}$, we have:

$$
\begin{aligned}
|(\mathbf{F x})(\mathrm{t})|= & \left|\frac{1}{\Gamma(\mathbf{q})} \int_{0}^{t}\left(e^{\mathrm{t}}-e^{s}\right)^{q-1} f(s, x(s)) e^{s} d s-\alpha \sum_{j=1}^{m} \gamma_{j} \int_{0}^{t_{j}}\left(e^{t_{j}}-e^{s}\right)^{q-1} f(s, x(s)) e^{s} d s\right| \\
\leqslant & \frac{1}{\Gamma(q)} \int_{0}^{t}\left(e^{t}-e^{s}\right)^{q-1}(|f(s, x(s))-f(s, 0)|+|f(s, 0)|) e^{s} d s \\
& +|\alpha| \sum_{j=1}^{m}\left|\gamma_{j}\right| \int_{0}^{t_{j}}\left(e^{t_{j}}-e^{s}\right)^{q-1}(|f(s, x(s))-f(s, 0)|+|f(s, 0)|) e^{s} d s \\
\leqslant & (\operatorname{Lr}+M) \frac{1}{\Gamma(q)}\left[\int_{0}^{t}\left(e^{t}-e^{s}\right)^{q-1} e^{s} d s+|\alpha| \sum_{j=1}^{m}\left|\gamma_{j}\right| \int_{0}^{t_{j}}\left(e^{t_{j}}-e^{s}\right)^{q-1} e^{s} d s\right] \\
\leqslant & (\operatorname{Lr}+M) \frac{1}{\Gamma(q+1)}\left[\left(e^{T}-1\right)^{q}+|\alpha|\left(e^{T}-1\right)^{q} \sum_{j=1}^{m}\left|\gamma_{j}\right|\right] \\
= & \frac{A\left(e^{T}-1\right)^{q}(\operatorname{Lr}+M)}{\Gamma(q+1)} \leqslant r .
\end{aligned}
$$

Now, for $x, y \in X$ and for each $t \in[0, T]$, we obtain

$$
\begin{aligned}
|(\mathbf{F} x)(\mathrm{t})-(\mathbf{F y})(\mathrm{t})| \leqslant & \frac{1}{\Gamma(\mathrm{q})} \int_{0}^{\mathrm{t}}\left(e^{\mathrm{t}}-e^{\mathrm{s}}\right)^{\mathrm{q}-1}|f(s, x(s))-f(s, y(s))| e^{s} d s \\
& +|\alpha| \sum_{j=1}^{\mathrm{m}}\left|\gamma_{j}\right| \frac{1}{\Gamma(\mathbf{q})} \int_{0}^{t_{j}}\left(e^{t_{j}}-e^{s}\right)^{q-1}|f(s, x(s))-f(s, y(s))| e^{s} d s d s \\
\leqslant & L\|x-y\| \frac{1}{\Gamma(q)}\left[\int_{0}^{t}\left(e^{t}-e^{s}\right)^{q-1} e^{s} d s+|\alpha| \sum_{j=1}^{m}\left|\gamma_{j}\right| \frac{1}{\Gamma(q)} \int_{0}^{t_{j}}\left(e^{t_{j}}-e^{s}\right)^{q-1} e^{s} d s\right] \\
\leqslant & \frac{L\left(e^{\top}-1\right)^{q} A}{\Gamma(q+1)}\|x-y\| .
\end{aligned}
$$

Since $\frac{L\left(e^{T}-1\right)^{q} A}{\Gamma(q+1)}<1$ it follows that $\mathbf{F}$ is a contraction. Thus, the conclusion of the theorem follows by the contraction mapping principle (Banach fixed point theorem). 
Now we establish an existence result for the nonlocal initial value problem (2.9), via Leray-Schauder nonlinear alternative.

Lemma 2.3 ([12, Nonlinear alternative for single valued maps]). Let $\mathrm{E}$ be a Banach space, $\mathrm{C}$ a closed, convex subset of $\mathrm{E}, \mathrm{U}$ an open subset of $\mathrm{C}$, and $0 \in \mathrm{U}$. Suppose that $\mathrm{F}: \overline{\mathrm{U}} \rightarrow \mathrm{C}$ is a continuous and compact (that is, $\mathrm{F}(\overline{\mathrm{U}})$ is a relatively compact subset of C) map. Then either

(i) F has a fixed point in $\overline{\mathrm{U}}$; or

(ii) there is $a \mathrm{u} \in \mathrm{\partial U}$ (the boundary of $\mathrm{U}$ in $\mathrm{C}$ ) and $\lambda \in(0,1)$ with $\mathrm{u}=\lambda \mathrm{F}(\mathrm{u})$.

Theorem 2.4. Let $\mathrm{f}:[0, \mathrm{~T}] \times \mathbb{R} \rightarrow \mathbb{R}$ be a continuous function. Assume that:

$\left(\mathrm{A}_{2}\right)$ There exist a function $\mathrm{p} \in \mathrm{L}^{1}\left([0, \mathrm{~T}], \mathbb{R}^{+}\right)$, and $\Omega: \mathbb{R}^{+} \rightarrow \mathbb{R}^{+}$nondecreasing such that $|\mathrm{f}(\mathrm{t}, \mathrm{x})| \leqslant$ $\mathrm{p}(\mathrm{t}) \Omega(\|x\|), \quad \forall(\mathrm{t}, \mathrm{x}) \in[0, \mathrm{~T}] \times \mathbb{R}$.

$\left(\mathrm{A}_{3}\right)$ There exists a constant $\mathrm{K}>0$ such that

$$
\frac{K}{A \Omega(K) \frac{1}{\Gamma(q)} \int_{0}^{T}\left(e^{T}-e^{s}\right)^{q-1} p(s) e^{s} d s}>1 .
$$

Then the initial value problem (2.1) has at least one solution on $[0, \mathrm{~T}]$.

Proof. We show the boundendness of the set of all solutions to equations $x=\lambda \mathbf{F} x$ for $\lambda \in[0,1]$, where $\mathbf{F}$ is defined by (2.5). Let $x$ be a solution of $x=\lambda \mathbf{F} x$ for $\lambda \in[0,1]$. Then for $t \in[0, T]$ we have

$$
\begin{aligned}
|x(t)|=|\lambda(\mathbf{F} x)(\mathrm{t})| & \leqslant \frac{1}{\Gamma(q)} \int_{0}^{t}\left(e^{t}-e^{s}\right)^{q-1} f(s, x(s)) e^{s} d s+|\alpha| \sum_{j=1}^{m}\left|\gamma_{j}\right| \frac{1}{\Gamma(q)} \int_{0}^{t_{j}}\left(e^{t_{j}}-e^{s}\right)^{q-1} f(s, x(s)) e^{s} d s \\
& \leqslant \frac{1}{\Gamma(q)} \int_{0}^{t}\left(e^{t}-e^{s}\right)^{q-1} p(s) \Omega(\|x\|) e^{s} d s+|\alpha| \sum_{j=1}^{m}\left|\gamma_{j}\right| \frac{1}{\Gamma(q)} \int_{0}^{t_{j}}\left(e^{t_{j}}-e^{s}\right)^{q-1} p(s) \Omega(\|x\|) e^{s} d s \\
& =A \Omega(\|x\|) \frac{1}{\Gamma(q)} \int_{0}^{T}\left(e^{T}-e^{s}\right)^{q-1} p(s) e^{s} d s,
\end{aligned}
$$

and consequently

$$
\frac{\|x\|}{A \Omega(\|x\|) \frac{1}{\Gamma(q)} \int_{0}^{T}\left(e^{T}-e^{s}\right)^{q-1} p(s) e^{s} d s} \leqslant 1 .
$$

In view of $\left(A_{3}\right)$, there is no solution $x$ such that $\|x\| \neq K$. Let us set

$$
\mathrm{U}=\{x \in \mathrm{X}:\|\mathrm{x}\|<\mathrm{K}\} .
$$

The operator $\mathbf{F}: \overline{\mathrm{U}} \rightarrow \mathrm{X}$ is continuous and completely continuous. Indeed, the continuity is obvious since $f$ is continuous, and for completely continuous we remark that it is uniformly bounded, since

$$
\begin{aligned}
|\mathbf{F} x(t)| & =\left|\frac{1}{\Gamma(q)} \int_{0}^{t}\left(e^{t}-e^{s}\right)^{q-1} f(s, x(s)) e^{s} d s-\alpha \sum_{j=1}^{m} \gamma_{j} \frac{1}{\Gamma(q)} \int_{0}^{t_{j}}\left(e^{t_{j}}-e^{s}\right)^{q-1} f(s, x(s)) e^{s} d s\right| \\
& \leqslant \frac{1}{\Gamma(q)} \int_{0}^{t}\left(e^{t}-e^{s}\right)^{q-1} p(s) \Omega(\|x\|) e^{s} d s+|\alpha| \sum_{j=1}^{m}\left|\gamma_{j}\right| \frac{1}{\Gamma(q)} \int_{0}^{T}\left(e^{T}-e^{s}\right)^{q-1} p(s) \Omega(\|x\|) e^{s} d s \\
& =A \Omega(r) \frac{1}{\Gamma(q)} \int_{0}^{T}\left(e^{T}-e^{s}\right)^{q-1} p(s) e^{s} d s
\end{aligned}
$$


and equicontinuous, since

$$
\begin{aligned}
\left|\mathbf{F} x\left(t_{2}\right)-\mathbf{F} x\left(t_{1}\right)\right|= & \mid \frac{1}{\Gamma(q)} \int_{0}^{t_{1}}\left[\left(e^{t_{2}}-e^{s}\right)^{q-1}-\left(e^{t_{1}}-e^{s}\right)^{q-1}\right] f(s, x(s)) e^{s} d s \\
& +\int_{t_{1}}^{t_{2}}\left(e^{t_{2}}-e^{s}\right)^{q-1} f(s, x(s)) e^{s} d s \mid \\
\leqslant & \mid \frac{\Omega(r)}{\Gamma(q)} \int_{0}^{t_{1}}\left[\left(e^{t_{2}}-e^{s}\right)^{q-1}-\left(e^{t_{1}}-e^{s}\right)^{q-1}\right] p(s) e^{s} d s \\
& +\frac{\Omega(r)}{\Gamma(q)} \int_{t_{1}}^{t_{2}}\left(e^{t_{2}}-e^{s}\right)^{q-1} p(s) e^{s} d s \mid .
\end{aligned}
$$

From the choice of $u$, there is no $u \in \partial u$ such that $u=\lambda \mathbf{F}(u)$ for some $\lambda \in(0,1)$. Consequently, by the nonlinear alternative of Leray-Schauder type (Lemma 2.3), we deduce that $F$ has a fixed point $u \in \bar{U}$ which is a solution of the problem (2.1). This completes the proof.

In the special case when $p(t)=1$ and $\Omega(\|x\|)=k\|x\|+N$, we have the following corollary.

Corollary 2.5. Let $\mathrm{f}:[0, \mathrm{~T}] \times \mathbb{R} \rightarrow \mathbb{R}$ be a continuous function. Assume that

$\left(\mathrm{A}_{4}\right)$ there exist constants $0 \leqslant k<\frac{\Gamma(\mathrm{q}+1)}{\mathrm{A}\left(\mathrm{e}^{\mathrm{T}}-1\right)^{\mathrm{q}}}$, and $\mathrm{N}>0$ such that $|\mathrm{f}(\mathrm{t}, \mathrm{x})| \leqslant \mathrm{k}|\mathrm{x}|+\mathrm{N}$ for all $\mathrm{t} \in[0, \mathrm{~T}], \mathrm{x} \in \mathbb{R}$.

Then the initial value problem (2.1) has at least one solution.

Example 2.6. Consider the following nonlinear exponential fractional differential equation with nonlocal (multi-point) initial condition of the form

$$
\left\{\begin{array}{l}
{ }_{C}^{e} D_{0}^{1 / 2} x(t)=f(t, x(t)), \quad 0<t<3 \\
x(0)+\frac{2}{3} x\left(\frac{1}{2}\right)-\frac{3}{4} x(1)+\frac{4}{5} x\left(\frac{3}{2}\right)-\frac{5}{6} x(2)+\frac{6}{7} x\left(\frac{5}{2}\right)=0 .
\end{array}\right.
$$

Here $\mathrm{q}=1 / 2, \mathrm{~T}=3, \mathrm{~m}=5, \gamma_{1}=2 / 3, \gamma_{2}=-3 / 4, \gamma_{3}=4 / 5, \gamma_{4}=-5 / 6, \gamma_{5}=6 / 7, \mathrm{t}_{1}=1 / 2, \mathrm{t}_{2}=1$, $t_{3}=3 / 2, t_{4}=2$, and $t_{5}=5 / 2$. So, we can check that $1+\sum_{j=1}^{5} \gamma_{j}=1.740476190 \neq 0$ and $A=3.244870042$. (i) Let the function $f$ is defined by

$$
f(t, x)=\frac{3 \sin ^{2} t}{(10+t)^{2}}\left(\frac{x^{2}+|x|}{1+|x|}\right)+\frac{1}{4}
$$

It is easy to compute that

$$
\frac{\Gamma(q+1)}{A\left(e^{T}-1\right)^{q}}=0.06251661312
$$

Since $|f(t, x)-f(t, y)| \leqslant(6 / 100)|x-y|$, we set a constant $L=3 / 50<0.06251661312$. Hence all conditions of Theorem 2.2 are satisfied. Therefore, the problem (2.6) with (2.7) has a unique solution.

(ii) Let the function $f$ is presented by

$$
f(t, x)=\frac{1}{1+t}\left(\frac{3 x^{6}}{10\left(1+|x|^{5}\right)}+\frac{1}{8}\right)
$$

By showing

$$
|f(t, x)| \leqslant \frac{1}{1+t}\left(\frac{3}{10}|x|+\frac{1}{8}\right),
$$

we set $p(t)=1 /(1+t)$ and $\Omega(u)=(3 u / 10)+(1 / 8)$ which leads to a constant $K>22.90686633$ satisfying inequality in $\left(\mathrm{A}_{3}\right)$. By applying Theorem 2.4, we get that the problem (2.6) with (2.8) has at least one solution on $[0,3]$. 


\subsection{Nonlocal boundary value problems}

In this subsection we study some nonlocal boundary value problems for exponential fractional differential equations. First we consider the following nonlocal boundary value problem

$$
\left\{\begin{array}{l}
{ }_{C}^{e} D_{0}^{\alpha} y(t)=f(t, y(t)), \quad t \in[0, T], \\
y(0)=g(y), \quad{ }^{e} D y(T)=h(y),
\end{array}\right.
$$

where ${ }_{C}^{e} D_{0}^{\alpha}$ is the exponential derivative of Caputo type which has fractional order $1<\alpha<2, f \in$ $\mathrm{C}([0, \mathrm{~T}] \times \mathbb{R}, \mathbb{R})$ and $\mathrm{g}, \mathrm{h}: \mathrm{C}([0, \mathrm{~T}], \mathbb{R}) \rightarrow \mathbb{R}$ are given continuous functions.

Lemma 2.7. The nonlocal boundary value problem (2.9) is equivalent to the following integral equation

$$
\begin{aligned}
y(t)= & g(y)+\left(e^{t}-1\right)\left(h(y)-\frac{1}{\Gamma(\alpha-1)} \int_{0}^{T}\left(e^{T}-e^{s}\right)^{\alpha-2} f(s, y(s)) e^{s} d s\right) \\
& +\frac{1}{\Gamma(\alpha)} \int_{0}^{t}\left(e^{t}-e^{s}\right)^{\alpha-1} f(s, y(s)) e^{s} d s .
\end{aligned}
$$

Proof. Applying the exponential fractional integral of order $\alpha$ to both sides of the equation in (2.9) and using Theorem 1.7, we obtain

$$
y(t)=c_{1}+\left(e^{t}-1\right) c_{2}+\frac{1}{\Gamma(\alpha)} \int_{0}^{t}\left(e^{t}-e^{s}\right)^{\alpha-1} f(s, y(s)) e^{s} d s
$$

Substituting boundary conditions, it follows that the integral equation (2.10) holds. On the other hand, by taking the exponential Caputo fractional derivative of order $\alpha$ to both sides of (2.10), we get ${ }_{C}^{e} D_{0}^{\alpha} y(t)=$ $f(t, y(t))$. Also, we have $y(0)=g(y)$ and

$$
{ }^{e} D y(t)=h(y)-\frac{1}{\Gamma(\alpha-1)} \int_{0}^{T}\left(e^{T}-s^{s}\right)^{\alpha-2} f(s, y(s)) e^{s} d s+\frac{1}{\Gamma(\alpha-1)} \int_{0}^{t}\left(e^{t}-e^{s}\right)^{\alpha-2} f(s, y(s)) e^{s} d s,
$$

from which we get ${ }^{e} \mathrm{Dy}(\mathrm{T})=\mathrm{h}(\mathrm{y})$. The proof is completed.

Theorem 2.8. Let $f:[0, T] \times \mathbb{R} \rightarrow \mathbb{R}$ be a continuous function. Assume that:

$\left(\mathrm{H}_{0}\right)$ there exists $\mathrm{L}>0$ such that

$$
|f(t, x)-f(t, y)| \leqslant L|x-y| \text { for } t \in[0, T] \text { and } x, y \in \mathbb{R} ;
$$

$\left(\mathrm{H}_{1}\right)$ there exist $k, \ell>0$ such that

$$
|g(x)-g(y)| \leqslant k|x-y|, \quad|h(x)-h(y)| \leqslant \ell|x-y| \text { for } x, y \in \mathbb{R} .
$$

If

$$
\mathrm{k}+\ell\left(e^{\top}-1\right)+\mathrm{L}(\alpha+1) \frac{\left(e^{\top}-1\right)^{\alpha}}{\Gamma(\alpha+1)}<1,
$$

then the nonlocal boundary value problem (2.9) has a unique solution on $[0, T]$.

Proof. We transform the boundary value problem (2.9) into the operator equation $y=A y$, where the operator $A: X \rightarrow X$ is defined by

$$
\begin{aligned}
A y(t)= & g(y)+\left(e^{t}-1\right)\left(h(y)-\frac{1}{\Gamma(\alpha-1)} \int_{0}^{T}\left(e^{T}-s^{s}\right)^{\alpha-2} f(s, y(s)) e^{s} d s\right) \\
& +\frac{1}{\Gamma(\alpha)} \int_{0}^{t}\left(e^{t}-e^{s}\right)^{\alpha-1} f(s, y(s)) e^{s} d s .
\end{aligned}
$$


Next, we use the Banach contraction principle to prove that the operator $A$ has a unique fixed point. To accomplish this we define a ball radius $r$ with

$$
r \geqslant \frac{|g(0)|+\left(e^{\top}-1\right)|h(0)|+\left(M(\alpha+1)\left(e^{\top}-1\right)^{\alpha} / \Gamma(\alpha+1)\right)}{1-\left(L(\alpha+1)\left(e^{\top}-1\right)^{\alpha} / \Gamma(\alpha+1)\right)-k-\ell\left(e^{\top}-1\right)}
$$

by $B_{r}=\{y \in X:\|y\| \leqslant r, r>0\}$. Now, let $\sup \{f(t, 0): t \in[0, T]\}=M$. For any $y \in B_{r}$, and using the fact that $|g(y)|=|g(y)-g(0)+g(0)| \leqslant k\|y\|+|g(0)|$ and $|h(y)| \leqslant \ell\|y\|+|h(0)|$, we have

$$
\begin{aligned}
|A y(t)| \leqslant & |g(y)|+\left(e^{T}-1\right)\left(|h(y)|+\frac{1}{\Gamma(\alpha-1)} \int_{0}^{T}\left(e^{T}-s^{s}\right)^{\alpha-2}(|f(s, y(s))-f(s, 0)|+|f(s, 0)|) e^{s} d s\right) \\
& +\frac{1}{\Gamma(\alpha)} \int_{0}^{t}\left(e^{t}-e^{s}\right)^{\alpha-1}(|f(s, y(s))-f(s, 0)|+|f(s, 0)|) e^{s} d s \\
\leqslant & k r+|g(0)|+\left(e^{T}-1\right)\left(\ell r+|h(0)|+(\operatorname{Lr}+M) \frac{\left(e^{T}-1\right)^{\alpha-1}}{\Gamma(\alpha)}\right)+(\operatorname{Lr}+M) \frac{\left(e^{T}-1\right)^{\alpha}}{\Gamma(\alpha+1)} \\
\leqslant & \left(k+\left(e^{T}-1\right) \ell\right) r+|g(0)|+\left(e^{T}-1\right)|h(0)|+(\alpha+1)(\operatorname{Lr}+M) \frac{\left(e^{T}-1\right)^{\alpha}}{\Gamma(\alpha+1)} \leqslant r,
\end{aligned}
$$

which implies that $A B_{r} \subset B_{r}$. Now, we show that the operator $A$ is a contraction operator. Let $x, y \in B_{r}$. Then we have

$$
\begin{aligned}
|A x(t)-A y(t)| \leqslant & |g(x)-g(y)|+\left(e^{T}-1\right)(|h(x)-h(y)| \\
& \left.+\frac{1}{\Gamma(\alpha-1)} \int_{0}^{T}\left(e^{T}-e^{s}\right)^{\alpha-2}|f(s, x(s))-f(s, y(s))| e^{s} d s\right) \\
& +\frac{1}{\Gamma(\alpha)} \int_{0}^{t}\left(e^{t}-e^{s}\right)^{\alpha-1}|f(s, x(s))-f(s, y(s))| e^{s} d s \\
\leqslant & k\|x-y\|+\left(e^{T}-1\right)\left(\ell\|x-y\|+L \frac{\left(e^{T}-1\right)^{\alpha-1}}{\Gamma(\alpha)}\|x-y\|\right)+L \frac{\left(e^{T}-1\right)^{\alpha}}{\Gamma(\alpha+1)}\|x-y\| \\
= & \left(k+\ell\left(e^{T}-1\right)+L(\alpha+1) \frac{\left(e^{T}-1\right)^{\alpha}}{\Gamma(\alpha+1)}\right)\|x-y\|,
\end{aligned}
$$

which yields that $\|A x-A y\| \leqslant\left(k+\ell\left(e^{\top}-1\right)+L(\alpha+1) \frac{\left(e^{T}-1\right)^{\alpha}}{\Gamma(\alpha+1)}\right)\|x-y\|$. From (2.11), the operator $A$ is a contraction which implies that $A$ has a unique fixed point by Banach's contraction principle. This, in turn, shows that the problem (2.9) has a unique solution on $[0, \mathrm{~T}]$. This completes the proof.

Now we establish an existence result for the nonlocal boundary value problem (2.9), via LeraySchauder nonlinear alternative.

Theorem 2.9. Let $\mathrm{f}: \mathrm{J} \times \mathbb{R} \rightarrow \mathbb{R}$ and $\mathrm{g}, \mathrm{h}: \mathrm{C}([0, \mathrm{~T}], \mathbb{R}) \rightarrow \mathbb{R}$ be continuous functions. Assume that $\left(\mathrm{H}_{1}\right)$ holds. In addition we suppose that:

$\left(\mathrm{H}_{2}\right)$ there exist a continuous nondecreasing function $\psi:[0, \infty) \rightarrow(0, \infty)$ and a function $p \in \mathrm{C}\left([0, \mathrm{~T}], \mathbb{R}^{+}\right)$such that

$$
|\mathrm{f}(\mathrm{t}, \mathrm{u})| \leqslant \mathrm{p}(\mathrm{t}) \psi(\|\mathrm{u}\|) \text { for each }(\mathrm{t}, \mathrm{u}) \in[0, \mathrm{~T}] \times \mathbb{R} ;
$$

$\left(\mathrm{H}_{3}\right)$ there exists a constant $\mathrm{M}>0$ such that

$$
\frac{\left[1-\left(k+\ell\left(e^{\top}-1\right)\right)\right] M}{|g(0)|+\left(e^{\top}-1\right)|h(0)|+(\alpha+1)\left(\|p\| \psi(M)\left(e^{\top}-1\right)^{\alpha} / \Gamma(\alpha+1)\right)}>1,
$$

with $k+\ell\left(e^{\top}-1\right)<1$. 
Then the nonlocal boundary value problem (2.9) has at least one solution on $[0, \mathrm{~T}]$.

Proof. We shall show that the operator $A: X \rightarrow X$ defined by (2.12) is continuous and completely continuous. Obviously $A$ is continuous since $f, g, h$ are continuous. We show that $A$ maps bounded sets into bounded sets in $X$. It is enough to show that for any $r>0$ there exists a positive constant $\tilde{\ell}$ such that for each $y \in B_{r}=\{y \in X:\|y\| \leqslant r\}$, we have $\|A y\| \leqslant \tilde{\ell}$. By $\left(H_{1}\right)$ and $\left(H_{2}\right)$, for each $t \in[0, T]$, we have

$$
\begin{aligned}
|A y(t)| \leqslant & k\|y\|+|g(0)|+\left(e^{T}-1\right)\left(\ell\|y\|+|h(0)|+\frac{\|p\| \psi(\|y\|)}{\Gamma(\alpha-1)} \int_{0}^{T}\left(e^{T}-e^{s}\right)^{\alpha-2} e^{s} d s\right) \\
& +\frac{\|p\| \psi(\|y\|)}{\Gamma(\alpha)} \int_{0}^{T}\left(e^{T}-e^{s}\right)^{\alpha-1} e^{s} d s \\
\leqslant & k\|y\|+|g(0)|+\left(e^{T}-1\right)\left(\ell\|y\|+|h(0)|+\frac{\|p\| \psi(\|y\|)\left(e^{T}-1\right)^{\alpha-1}}{\Gamma(\alpha)}\right)+\frac{\|p\| \psi(\|y\|)\left(e^{T}-1\right)^{\alpha}}{\Gamma(\alpha+1)} \\
\leqslant & \left(k+\ell\left(e^{\top}-1\right)\right) r+|g(0)|+\left(e^{T}-1\right)|h(0)|+(\alpha+1) \frac{\|p\| \psi(r)\left(e^{\top}-1\right)^{\alpha}}{\Gamma(\alpha+1)} .
\end{aligned}
$$

Thus

$$
\|A y\| \leqslant\left(k+\ell\left(e^{\top}-1\right)\right) r+|g(0)|+\left(e^{\top}-1\right)|h(0)|+(\alpha+1) \frac{\|p\| \psi(r)\left(e^{\top}-1\right)^{\alpha}}{\Gamma(\alpha+1)}:=\tilde{\ell} .
$$

Next we show that $A$ maps bounded sets into equicontinuous sets of $X$. Let $t_{1}, t_{2} \in[0, T]$ with $t_{1}<t_{2}$, $B_{r}$ be a bounded set of $X$ as above. Then, for any $y \in B_{r}$, we have

$$
\begin{aligned}
\left|A y\left(t_{2}\right)-A y\left(t_{1}\right)\right| \leqslant & \left|e^{t_{2}}-e^{t_{1}}\right|\left(\ell r+|h(0)|+\frac{\|p\| \psi(r)\left(e^{T}-1\right)^{\alpha-1}}{\Gamma(\alpha)}\right) \\
& +\frac{1}{\Gamma(\alpha)} \int_{0}^{t_{1}}\left[\left(e^{t_{2}}-e^{s}\right)^{\alpha-1}-\left(e^{t_{1}}-e^{s}\right)^{\alpha-1}\right]|f(s, y(s))| e^{s} d s \\
& +\frac{1}{\Gamma(\alpha)} \int_{t_{1}}^{t_{2}}\left(e^{t_{2}}-e^{s}\right)^{\alpha-1}|f(s, y(s))| e^{s} d s \\
\leqslant & \mid e^{t_{2}}-e^{t_{1} \mid}\left(\ell r+|h(0)|+\frac{\|p\| \psi(r)\left(e^{T}-1\right)^{\alpha-1}}{\Gamma(\alpha)}\right) \\
& +\frac{\|p\| \psi(r)}{\Gamma(\alpha+1)}\left\{\int_{0}^{t_{1}}\left[\left(e^{t_{2}}-e^{s}\right)^{\alpha-1}-\left(e^{t_{1}}-e^{s}\right)^{\alpha-1}\right] e^{s} d s+\int_{t_{1}}^{t_{2}}\left|\left(e^{t_{2}}-e^{s}\right)^{\alpha-1}\right| e^{s} d s\right\} \\
\leqslant & \mid e^{t_{2}}-e^{t_{1} \mid}\left(\ell r+|h(0)|+\frac{\|p\| \psi(r)\left(e^{T}-1\right)^{\alpha-1}}{\Gamma(\alpha)}\right) \\
& +\frac{\|p\| \psi(r)}{\Gamma(\alpha+1)}\left\{2\left(e^{t_{2}}-e^{t_{1}}\right)^{\alpha}+\left(e^{t_{1}}-1\right)^{\alpha}-\left(e^{t_{2}}-1\right)^{\alpha}\right\} .
\end{aligned}
$$

As $t_{1} \longrightarrow t_{2}$, the right-hand side of the above inequality tends to zero. In consequence, the Arzelá-Ascoli theorem follows that $A: X \longrightarrow X$ is continuous and completely continuous.

Finally, we show that there exists an open set $U \subseteq X$ with $y \neq \lambda A y$ for $\lambda \in(0,1)$ and $y \in \partial U$.

Let $y \in X$ and $y=\lambda A(y)$ for some $0<\lambda<1$. Then, for each $t \in J$, we have by the computations above

$$
\|y\| \leqslant\left(k+\ell\left(e^{\top}-1\right)\right)\|y\|+|g(0)|+\left(e^{\top}-1\right)|h(0)|+(\alpha+1) \frac{\|p\| \psi(\|y\|)\left(e^{\top}-1\right)^{\alpha}}{\Gamma(\alpha+1)},
$$

or

$$
\frac{\left[1-\left(k+\ell\left(e^{\top}-1\right)\right)\right]\|y\|}{|g(0)|+\left(e^{\top}-1\right)|h(0)|+(\alpha+1)\left(\|p\| \psi(\|y\|)\left(e^{\top}-1\right)^{\alpha} / \Gamma(\alpha+1)\right)} \leqslant 1 .
$$


In view of $\left(\mathrm{H}_{3}\right)$, there exists $M$ such that $\|y\| \neq M$. Let us set

$$
\mathrm{U}=\{\mathrm{y} \in \mathrm{X}:\|\mathrm{y}\|<\mathrm{M}\}
$$

Note that the operator $A: \bar{U} \rightarrow X$ is continuous and completely continuous. From the choice of $U$, there is no $y \in \partial U$ such that $y=\lambda A y$ for some $\lambda \in(0,1)$. Consequently, by the nonlinear alternative of LeraySchauder type (Lemma 2.3), we deduce that $A$ has a fixed point $y \in \bar{U}$ which is a solution of the problem (2.9). This completes the proof.

The final existence result is based on Krasnoselskii's fixed point theorem [14].

Lemma 2.10 ([14, Krasnoselskii's fixed point theorem]). Let $\mathrm{S}$ be a closed, convex, and nonempty subset of a Banach space X. Let $A, B$ be the operators such that (a) $A x+B y \in S$ whenever $x, y \in S$; (b) $A$ is compact and continuous; (c) B is a contraction mapping. Then there exists $z \in \mathrm{S}$ such that $z=\mathrm{A} z+\mathrm{B} z$.

Theorem 2.11. Assume that $\left(\mathrm{H}_{0}\right)$ holds. In addition we assume that:

$\left(\mathrm{H}_{4}\right) \quad|\mathrm{f}(\mathrm{t}, \mathrm{y})| \leqslant \mu(\mathrm{t}), \quad|\mathrm{g}(\mathrm{y})| \leqslant \lambda(\mathrm{t}), \quad|\mathrm{h}(\mathrm{y})| \leqslant v(\mathrm{t}), \quad$ and $\mu, \lambda, v \in \mathrm{C}\left([0, \mathrm{~T}], \mathbb{R}^{+}\right)$.

Then the nonlocal boundary problem (2.9) has at least one solution on $[0, \mathrm{~T}]$, provided

$$
k+\ell\left(e^{\top}-1\right)+L \frac{\left(e^{\top}-1\right)^{\alpha}}{\Gamma(\alpha)}<1
$$

Proof. To apply Lemma 2.10, we define the operators P and Q by

$$
P y(t)=g(y)+\left(e^{t}-1\right)\left(h(y)-\frac{1}{\Gamma(\alpha-1)} \int_{0}^{T}\left(e^{T}-s^{s}\right)^{\alpha-2} f(s, y(s)) e^{s} d s\right)
$$

and

$$
\mathrm{Qy}(\mathrm{t})=\frac{1}{\Gamma(\alpha)} \int_{0}^{\mathrm{t}}\left(e^{\mathrm{t}}-\mathrm{e}^{\mathrm{s}}\right)^{\alpha-1} \mathrm{f}(\mathrm{s}, \mathrm{y}(\mathrm{s})) \mathrm{e}^{\mathrm{s}} \mathrm{ds}, \mathrm{t} \in[0, \mathrm{~T}] .
$$

Setting $\sup _{t \in[0, T]}|\mu(t)|=\|\mu\|, \sup _{t \in[0, T]}|\lambda(t)|=\|\lambda\|, \sup _{t \in[0, T]}|v(t)|=\|v\|$, and choosing a constant

$$
\rho \geqslant\|\lambda\|+\left(e^{\top}-1\right)\|v\|+\|\mu\|(\alpha+1) \frac{\left(e^{\top}-1\right)^{\alpha}}{\Gamma(\alpha+1)},
$$

we define a ball $B_{\rho}=\{y \in C(J, \mathbb{R}):\|y\| \leqslant \rho\}$. For any $y, z \in B_{\rho}$, we have

$$
\begin{aligned}
|\mathrm{Py}(\mathrm{t})+\mathrm{Q} z(\mathrm{t})| & \leqslant|g(y)|+\left(e^{\top}-1\right)\left(|\mathrm{h}(\mathrm{y})|+\|\mu\| \frac{\left(e^{\top}-1\right)^{\alpha-1}}{\Gamma(\alpha)}\right)+\|\mu\| \frac{\left(e^{\top}-1\right)^{\alpha}}{\Gamma(\alpha+1)} \\
& \leqslant\|\lambda\|+\left(e^{\top}-1\right)\|v\|+\|\mu\|(\alpha+1) \frac{\left(e^{\top}-1\right)^{\alpha}}{\Gamma(\alpha+1)} \leqslant \rho .
\end{aligned}
$$

Hence

$$
\|\mathrm{Py}+\mathrm{Q} z\| \leqslant \rho,
$$

which shows that $\mathrm{Py}+\mathrm{Q} z \in \mathrm{B}_{\rho}$. It is easy to see using (2.13) that $\mathrm{P}$ is a contraction mapping.

Continuity of $f$ implies that the operator $Q$ is continuous. Also, $Q$ is uniformly bounded on $B_{\rho}$ as

$$
\|\mathrm{Q} y\| \leqslant\|\mu\| \frac{\left(e^{\top}-1\right)^{\alpha}}{\Gamma(\alpha+1)}
$$

Also $\mathrm{Q}$ is equicontinuous, as proved in Theorem 2.9. So $\mathrm{Q}$ is relatively compact on $\mathrm{B}_{\rho}$. Hence, by the Arzelá-Ascoli theorem, $\mathrm{Q}$ is compact on $\mathrm{B}_{\rho}$. Thus all the assumptions of Lemma 2.10 are satisfied. So the conclusion of Lemma 2.10 implies that the problem (2.9) has at least one solution on $[0, \mathrm{~T}]$. 
Example 2.12. Consider the following nonlinear exponential fractional differential equation with nonlocal boundary condition of the form

$$
\left\{\begin{array}{l}
{ }_{C}^{e} D_{0}^{3 / 2} y(t)=f(t, y(t)), \quad 0<t<1, \\
y(0)=g(y), \quad{ }^{e} D y(T)=h(y) .
\end{array}\right.
$$

Here $q=3 / 2$ and $T=1$. Next we give some cases of nonlinear function and nonlocal boundary conditions.

(i) Let the functions $f, g$, and $h$ are defined by

$$
f(t, y)=\frac{1}{10(\sqrt{t}+3)}\left(\frac{y^{2}+6|y|}{5+|y|}\right)+\frac{1}{2}, g(y)=\frac{|y(1 / 2)|}{3+|y(1 / 2)|}, h(y)=\frac{1}{6} \sin \left(y\left(\frac{2}{3}\right)\right)
$$

Then we have

$$
|f(t, x)-f(t, y)| \leqslant \frac{1}{15}|x-y|, \quad|g(x)-g(y)| \leqslant \frac{1}{3}|x-y|, \text { and }|h(x)-h(y)| \leqslant \frac{1}{6}|x-y|, \forall x, y \in \mathbb{R} .
$$

Setting $L=1 / 15, k=1 / 3$, and $\ell=1 / 6$, we see that

$$
k+\ell\left(e^{\top}-1\right)+L(\alpha+1) \frac{\left(e^{\top}-1\right)^{\alpha}}{\Gamma(\alpha+1)}=0.9021067798<1 .
$$

By applying Theorem 2.8, the problem (2.14) with (2.15) has a unique solution.

(ii) Let the given functions $f, g$, and $h$ are defined as

$$
f(t, y)=\left(\frac{1}{t^{2}+3}\right)\left(\frac{y^{8}}{6\left(1+|y|^{7}\right)}+\frac{2}{3}\right), g(y)=\frac{1}{4} \tan ^{-1}\left(y\left(\frac{1}{3}\right)\right)+\frac{4}{3}, h(y)=\frac{|y(1 / 8)|}{7(1+|y(1 / 8)|)}+\frac{1}{5} .
$$

From the above information, we get

$$
|f(t, y)| \leqslant\left(\frac{1}{t^{2}+3}\right)\left(\frac{1}{6}|y|+\frac{2}{3}\right),
$$

and $|g(x)-g(y)| \leqslant(1 / 4)|x-y|$ and $|h(x)-h(y)| \leqslant(1 / 7)|x-y|$. Then we choose $p(t)=1 /\left(t^{2}+3\right)$, $\psi(u)=(1 / 6) u+(2 / 3), k=1 / 4, \ell=1 / 7, g(0)=4 / 3, h(0)=1 / 5$. Consequently, we can find that there exists a constant $M>9.726098262$ satisfying the inequality in condition $\left(\mathrm{H}_{3}\right)$. By mean of Theorem 2.9, we have that the problem (2.14) with (2.16) has at least one solution on $[0,1]$.

(iii) Let the functions $f, g$, and $h$ are given by

$$
f(t, y)=\frac{e^{-t}}{8+t^{3}}|\sin y|+\frac{1}{4}, \quad g(y)=\frac{|y(1 / 4)|}{3(1+|y(1 / 4)|)}+\frac{1}{3}, \text { and } h(y)=\frac{5}{12}+\frac{1}{6} \tan ^{-1} y\left(\frac{5}{6}\right) .
$$

Now, we can show that

$$
|f(t, y)| \leqslant \frac{e^{-t}}{8+t^{3}}+\frac{1}{4}, \quad|g(y)| \leqslant \frac{2}{3}, \quad h(y) \leqslant \frac{5+\pi}{12}
$$

and also $|f(t, x)-f(t, y)| \leqslant(1 / 8)|x-y|,|g(x)-g(y)| \leqslant(1 / 3)|x-y|$ and $|h(x)-h(y)| \leqslant(1 / 6)|x-y|$. It follows that

$$
k+\ell\left(e^{\top}-1\right)+L \frac{\left(e^{\top}-1\right)^{\alpha}}{\Gamma(\alpha)}=0.9374059226<1 .
$$

This implies by Theorem 2.11 that the problem (2.14) with (2.17) has at least one solution on $[0,1]$. 


\section{Remarks}

Many other boundary value problems can be studied by using the methods of this paper. For example, let us consider the following boundary value problem

$$
\left\{\begin{array}{l}
{ }_{C}^{e} D_{0}^{\alpha} y(t)=f(t, y(t)), \quad t \in[0, T] \\
y(0)=g(y), \quad{ }^{e} D y(T)=\lambda y(\eta), \quad \eta \in(0, T),
\end{array}\right.
$$

where ${ }_{C}^{e} D_{0}^{\alpha}$ is the exponential derivative of Caputo type which has fractional order $1<\alpha<2, f \in$ $\mathrm{C}([0, \mathrm{~T}] \times \mathbb{R}, \mathbb{R}), \mathrm{g}: \mathrm{C}([0, \mathrm{~T}], \mathbb{R}) \rightarrow \mathbb{R}$ is a given continuous function and $\lambda$ a real constant with $\lambda \neq$ $\left(e^{\eta}-1\right)^{-1}$. This is a special case of the problem (2.9) with $h(y)=\lambda y(\eta)$.

Lemma 3.1. The nonlocal boundary value problem (3.1) is equivalent to the following integral equation

$$
\begin{aligned}
y(t)= & g(y)+\frac{\left(e^{t}-1\right)}{1-\lambda\left(e^{\eta}-1\right)}\left(\lambda g(y)+\frac{\lambda}{\Gamma(\alpha)} \int_{0}^{\eta}\left(e^{\eta}-e^{s}\right)^{\alpha-1} f(s, y(s)) e^{s} d s\right. \\
& \left.-\frac{1}{\Gamma(\alpha-1)} \int_{0}^{T}\left(e^{T}-e^{s}\right)^{\alpha-2} f(s, y(s)) e^{s} d s\right)+\frac{1}{\Gamma(\alpha)} \int_{0}^{t}\left(e^{t}-e^{s}\right)^{\alpha-1} f(s, y(s)) e^{s} d s .
\end{aligned}
$$

We include without proofs the results.

Theorem 3.2. Let $\mathrm{f}:[0, \mathrm{~T}] \times \mathbb{R} \rightarrow \mathbb{R}$ be a continuous function. Assume that $\left(\mathrm{H}_{0}\right)$ and $\left(\mathrm{H}_{1}\right)$ hold. If

$$
k+\frac{e^{\top}-1}{\left|1-\lambda\left(e^{\eta}-1\right)\right|}\left\{\lambda k+L \lambda \frac{\left(e^{\eta}-1\right)^{\alpha}}{\Gamma(\alpha+1)}+L \frac{\left(e^{\top}-1\right)^{\alpha-1}}{\Gamma(\alpha)}\right\}+L \frac{\left(e^{\top}-1\right)^{\alpha}}{\Gamma(\alpha+1)}<1,
$$

then the nonlocal boundary value problem (3.1) has a unique solution on $[0, \mathrm{~T}]$.

Theorem 3.3. Let $\mathrm{f}: \mathrm{J} \times \mathbb{R} \rightarrow \mathbb{R}$ and $\mathrm{g}, \mathrm{h}: \mathrm{C}([0, \mathrm{~T}], \mathbb{R}) \rightarrow \mathbb{R}$ be continuous functions. Assume that $\left(\mathrm{H}_{1}\right)$ and $\left(\mathrm{H}_{2}\right)$ hold. In addition we suppose that:

$\left(\mathrm{H}_{3}\right)^{\prime}$ there exists a constant $\mathrm{M}>0$ such that

$$
\frac{\mathrm{BM}}{\mathrm{A}_{1}|g(0)|+\mathrm{A}_{2}\|p\| \psi(M)}>1,
$$

where

$$
\begin{gathered}
B=1-k-k|\lambda| \frac{\left(e^{\top}-1\right)}{\left|1-\lambda\left(e^{\eta}-1\right)\right|}>0, \quad A_{1}=1+\frac{|\lambda|\left(e^{\top}-1\right)}{\left|1-\lambda\left(e^{\eta}-1\right)\right|^{\prime}} \\
A_{2}=\frac{\left(e^{\top}-1\right)}{\left|1-\lambda\left(e^{\eta}-1\right)\right|} \frac{|\lambda|\left(e^{\eta}-1\right)^{\alpha}}{\Gamma(\alpha+1)}+\frac{\left(e^{\top}-1\right)^{\alpha}}{\Gamma(\alpha)\left|1-\lambda\left(e^{\eta}-1\right)\right|}+\frac{\left(e^{\top}-1\right)^{\alpha}}{\Gamma(\alpha+1)} .
\end{gathered}
$$

Then the nonlocal boundary value problem (3.1) has at least one solution on $[0, \mathrm{~T}]$.

Theorem 3.4. Assume that $\left(\mathrm{H}_{0}\right)$ and $\left(\mathrm{H}_{4}\right)$ hold. Then the nonlocal boundary value problem (3.1) has at least one solution on $[0, \mathrm{~T}]$, provided

$$
k+\frac{e^{\top}-1}{\left|1-\lambda\left(e^{\eta}-1\right)\right|}\left\{\lambda k+L \frac{\left(e^{\eta}-1\right)^{\alpha}}{\Gamma(\alpha+1)}+\mathrm{L} \frac{\left(e^{\top}-1\right)^{\alpha-1}}{\Gamma(\alpha)}\right\}<1 .
$$


As a second example, we consider the following boundary value problem with nonlocal boundary conditions

$$
\left\{\begin{array}{l}
{ }_{C}^{e} D_{0}^{\alpha} y(t)=f(t, y(t)), \quad t \in[0, T], \\
y(0)=g(y), \quad y(T)=\sum_{i=1}^{n} \gamma_{i} y\left(\xi_{i}\right), \quad \xi_{i} \in(0, T),
\end{array}\right.
$$

where ${ }_{C}^{e} D_{0}^{\alpha}$ is the exponential derivative of Caputo type which has fractional order $1<\alpha<2, f \in$ $\mathrm{C}([0, T] \times \mathbb{R}, \mathbb{R}), \mathrm{g}: \mathrm{C}([0, \mathrm{~T}], \mathbb{R}) \rightarrow \mathbb{R}$ is a given continuous function, and $\gamma_{i}, i=1,2, \ldots, n$ are real constants with $\sum_{i=1}^{n} \gamma_{i}\left(e^{\xi_{i}}-1\right) \neq e^{\top}-1$.

In the next lemma we give only the expression of the solution. The results can be obtained as in the previous boundary value problems and are omitted.

Lemma 3.5. Assume that $\Lambda:=e^{\top}-1-\sum_{i=1}^{n} \gamma_{i}\left(e^{\xi_{i}}-1\right) \neq 0$. Then the nonlocal boundary value problem (3.2) is equivalent to the following integral equation

$$
\begin{aligned}
y(t)= & g(y)+\frac{\left(e^{t}-1\right)}{\Lambda}\left\{\sum_{i=1}^{n} \gamma_{i}\left[g(y)+\frac{1}{\Gamma(\alpha)} \int_{0}^{\xi_{i}}\left(e^{\xi_{i}}-e^{s}\right)^{\alpha-1} f(s, y(s)) e^{s} d s\right]\right. \\
& \left.-g(y)-\frac{1}{\Gamma(\alpha)} \int_{0}^{T}\left(e^{T}-e^{s}\right)^{\alpha-1} f(s, y(s)) e^{s} d s\right\}+\frac{1}{\Gamma(\alpha)} \int_{0}^{t}\left(e^{t}-e^{s}\right)^{\alpha-1} f(s, y(s)) e^{s} d s .
\end{aligned}
$$

\section{References}

[1] R. P. Agarwal, Y. Zhou, Y. Y. He, Existence of fractional neutral functional differential equations, Comput. Math. Appl., 59 (2010), 1095-1100. 1

[2] R. P. Agarwal, Y. Zhou, J. R. Wang, X. N. Luo, Fractional functional differential equations with causal operators in Banach spaces, Math. Comput. Modelling, 54 (2011), 1440-1452.

[3] B. Ahmad, S. K. Ntouyas, Nonlinear fractional differential equations and inclusions of arbitrary order and multi-strip boundary conditions, Electron. J. Differential Equations, 2012 (2012), 22 pages.

[4] B. Ahmad, S. K. Ntouyas, Integro-differential equations of fractional order with nonlocal fractional boundary conditions associated with financial asset model, Electron. J. Differential Equations, 2013 (2013), 10 pages.

[5] B. Ahmad, S. K. Ntouyas, A. Alsaedi, New existence results for nonlinear fractional differential equations with three-point integral boundary conditions, Adv. Difference Equ., 2011 (2011), 11 pages.

[6] B. Ahmad, S. Sivasundaram, On four-point nonlocal boundary value problems of nonlinear integro-differential equations of fractional order, Appl. Math. Comput., 217 (2010), 480-487. 1

[7] D. R. Anderson, A. Boucherif, Nonlocal initial value problem for first-order dynamic equations on time scales, Dyn. Contin. Discrete Impuls. Syst. Ser. A Math. Anal., 16 (2009), 222-226. 2.1

[8] A. Boucherif, R. Precup, On the nonlocal initial value problem for first order differential equations, Fixed Point Theory, 4 (2003), 205-212. 2.1

[9] A. Boucherif, S. K. Ntouyas, Nonlocal initial value problems for first order fractional differential equations, Dynam. Systems Appl., 20 (2011), 247-259. 2.1

[10] K. Diethelm, The Analysis of Fractional Differential Equations, Springer-verlag, Berlin, (2010). 1

[11] A. M. A. El-Sayed, Nonlinear functional differential equations of arbitrary orders, Nonlinear Anal., 33 (1998), 181-186. 1

[12] A. Granas, J. Dugundji, Fixed Point Theory, Springer-Verlag, New York, (2003). 2.3

[13] A. A. Kilbas, H. M. Srivastava, J. J. Trujillo, Theory and Applications of Fractional Differential Equations, Elsevier Science B.V., Amsterdam, (2006). 1

[14] M. A. Krasnoselskii, Two remarks on the method of successive approximations, Uspekhi Mat. Nauk, 10 (1955), $123-127$. 2.2, 2.10

[15] V. Lakshmikantham, S. Leela, J. V. Devi, Theory of Fractional Dynamic Systems, Cambridge Academic Publishers, Cambridge, (2009). 1

[16] K. S. Miller, B. Ross, An Introduction to the Fractional Calculus and Differential Equations, John Wiley \& Sons, New York, (1993). 1

[17] S. Niyom, S. K. Ntouyas, S. Laoprasittichok, J. Tariboon, Boundary value problems with four orders of Riemann-Liouville fractional derivatives, Adv. Difference Equ., 2016 (2016), 14 pages. 1

[18] S. K. Ntouyas, J. Tariboon, C. Thaiprayoon, Nonlocal boundary value problems for Riemann-Liouville fractional differential inclusions with Hadamard fractional integral boundary conditions, Taiwanese J. Math., 20 (2016), 91-107. 1 
[19] I. Podlubny, Fractional Differential Equations, Academic Press, San Diego, (1999). 1

[20] S. G. Samko, A. A. Kilbas, O. I. Marichev, Fractional Integrals and Derivatives: Theory and Applications, Gordon and Breach Science Publishers, Yverdon, (1993). 1

[21] J. Tariboon, S. K. Ntouyas, P. Thiramanus, Riemann-Liouville fractional differential equations with Hadamard fractional integral conditions, Inter. J. Appl. Math. Stat., 54 (2016), 119-134. 1

[22] J. Tariboon, S. K. Ntouyas, W. Sudsutad, Fractional integral problems for fractional differential equations via Caputo derivative, Adv. Difference Equ., 2014 (2014), 17 pages. 1

[23] J. Tariboon, S. K. Ntouyas, Initial and boundary value problems via exponential fractional calculus, International Electronic Journal of Pure and Applied Mathematics 12 (2018), 1-19. 1

[24] C. Yu, G. Z. Gao, Some results on a class of fractional functional differential equations, Comm. Appl. Nonlinear Anal., 11 (2004), 67-75. 1

[25] C. Yu, G. Z. Gao, Existence of fractional differential equations, J. Math. Anal. Appl., 310 (2005), 26-29. 1 